\title{
Postauricular percutaneous power delivery for permanent mechanical circulatory support
}

\author{
Stephen Westaby, PhD, FRCS, MS \\ Robert Jarvik, $M D^{\mathrm{b}}$ \\ Andrew Freeland, FRCS ${ }^{\mathrm{d}}$ \\ David Pigott, FRCA ${ }^{\text {a }}$ \\ Desiree Robson, $\mathrm{RN}^{\mathrm{a}}$ \\ Satoshi Saito, MD, $\mathrm{PhD}^{\mathrm{a}}$ \\ Pedro Catarino, FRCS ${ }^{\mathrm{C}}$ \\ 0. H. Frazier, $M^{c}$
}

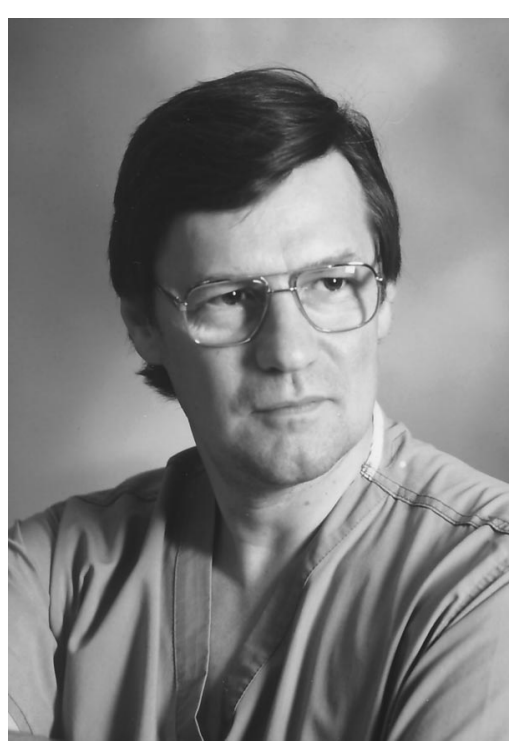

Dr Westaby

From the Oxford Heart Centre, ${ }^{\text {a }}$ Oxford and The Heart Hospital, London, United Kingdom; Jarvik Heart Corp, ${ }^{\text {b New York, }}$ NY; Texas Heart Institute, ${ }^{c}$ Houston, Tex; and Department of Otolaryngology, ${ }^{\mathrm{d}}$ Radcliffe Infirmary, Oxford, United Kingdom.

Supported by charitable donations from the National Heart Research Fund and the Artificial Heart Fund, UK. Robert Jarvik, MD, is the chief executive of Jarvik Heart Inc. None of the other investigators has a financial interest in the company.

Address for reprints: Stephen Westaby, $\mathrm{PhD}$, FRCS, MS, Oxford Heart Centre, John Radcliffe Hospital, Headington, Oxford OX3 9DU, United Kingdom (E-mail: swestaby@AHF.org.uk).

J Thorac Cardiovasc Surg 2002;123:977-83

Copyright $\odot 2002$ by The American Association for Thoracic Surgery

0022-5223/2002 \$35.00+0 $\quad \mathbf{1 2 / 1 / 1 2 1 0 4 5}$

doi: $10.1067 / \mathrm{mtc} .2002 .121045$
Objective: Percutaneous driveline infection continues to detract from both quality and length of life in patients with a left ventricular assist device. We have pursued an alternative route by using a skull-mounted percutaneous pedestal similar to cochlear implant technology. We have now used this method in patients implanted with the Jarvik 2000 heart (Jarvik Heart, Inc, New York, NY) as destination therapy for end-stage (New York Heart Association class IV) heart failure.

Methods: Four men with cardiomyopathy aged 61 to 72 years received the Jarvik 2000 heart with postauricular power delivery for permanent mechanical circulatory support. The power cable was brought through the second posterior intercostal space and routed through the neck to a percutaneous titanium implant screwed to the skull. This joins with the cable to the external controller and battery.

Results: In 3 patients the pedestal healed well and remained free from infection up to 1 year. The system was user friendly, and the whole external apparatus is exchangeable. The second patient had a subdural hematoma. This caused us to improve the preparation and modify the implant procedure.

Conclusion: For widespread use, permanent implantable circulatory support requires a reliable, user-friendly device with freedom from powerline infection. Our early experience with the Jarvik 2000 heart suggests that rigid fixation and the vascularity of scalp skin promote healing and reduce the risk of driveline infection.

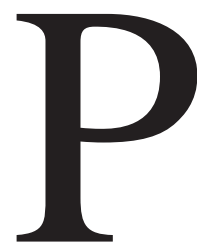

ercutaneous driveline infection continues to detract from both quality and length of life in patients undergoing placement of a left ventricular assist device (LVAD). ${ }^{1,2}$ Now that implantable axial flow and centrifugal blood pumps provide the prospect for permanent mechanical circulatory support, it is important to minimize the infection risk. Transcutaneous energy transfer with induction coils is an emerging option but requires a substantial increase in the implantable components attached to the blood pump. ${ }^{3}$ We have pursued an alternative route by using a skull-mounted percutaneous pedestal similar to cochlear implant technology (Figure 1). ${ }^{4}$ In laboratory testing this innovation provided long-term freedom from infection through rigid fixation, absence of subcutaneous fat, and efficient healing of highly vascular scalp skin. ${ }^{5}$ We have now applied this technology in patients implanted with the Jarvik 2000 heart (Jarvik Heart, Inc, New York, NY) as destination therapy for end-stage heart failure. ${ }^{6}$ This article describes our learning curve and the pitfalls and advantages of the procedure. 


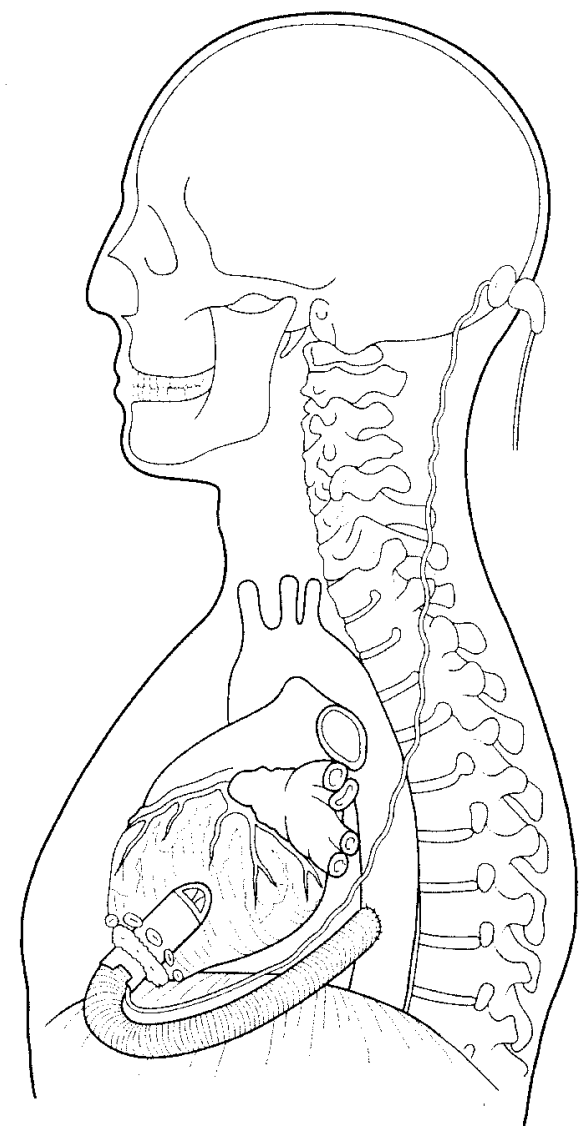

Figure 1. Diagram showing the principle of power delivery to the Jarvik 2000 heart.

\section{Patients and Methods}

Four male patients aged 61 to 72 years with end-stage (New York Heart Association class IV) dilated cardiomyopathy have received the Jarvik 2000 heart with postauricular percutaneous power delivery in a limited clinical trial of permanent implantable circulatory support. The trial was approved by the Medical Device Agency (United Kingdom) and the ethics committees of the Oxford Radcliffe Hospital Trust and The Heart Hospital, London. It is now 1 year since implantation in the first patient.

\section{Device and Power System}

The Jarvik 2000 heart is implanted into the apex of the failing left ventricle, from which a $16-\mathrm{mm}$ impervious Dacron graft conveys blood to the descending thoracic aorta (Figure 1). ${ }^{6}$ The loosely braided flexible power cable is composed of a bundle of 9 individually insulated pacemaker-type leads, which are extrusion insulated with polycarbonate polyurethane. The internal cable (Figure 2) terminates in a connector within the percutaneous titanium pedestal (Figure 3). The external power cable, which can be replaced in the event of damage, connects from the skull-mounted pedestal to the belt-worn control system, which in turn is connected to the battery (Figure 4). The cable uses a special connector that plugs directly into the skull-mounted pedestal and is keyed so that it cannot be connected improperly. The pump is controlled by the external all-analogue control system in which the motor pump speed is set manually and maintained at the set level by a pulse width, modulated, speed-control circuit. The control unit incorporates a watt meter, which displays power use and alarm functions for the pump under speed, low battery reserve, or circuit failure. The belt-worn lithium ion (Li-ion) battery provides more than 8 hours of support at nominal flows of 3 to $4 \mathrm{~L} / \mathrm{min}$ under normotensive conditions. At higher flow or pressure, the belt-worn packs discharge faster. The controller and battery system weighs only 1 $\mathrm{kg}$.

\section{Preparation for the Postauricular Pedestal}

A computed tomographic (CT) scan of the skull is obtained, followed by a 3-dimensional reconstruction to measure skull thickness in the parietal area. This is variable (usually between 5 and 7 $\mathrm{mm}$ ), and it is important that the screws do not penetrate the inner table. Two days before the operation, the hair is cut short, and the postauricular aspect of the scalp is shaved on the left side. The site of the pedestal and exit site from the chest are identified and marked with an indelible pen, with the patient in the sitting position. The site of exit from the chest is one handsbreadth from the midline and $2 \mathrm{~cm}$ above the top of the scapula in the sitting position. The incision passes downward into the second posterior intercostal space without danger of damaging either blood vessels or nerves.

The pedestal is attached to the parietal bone about $5 \mathrm{~cm}$ behind and slightly above the ear (Figure 2). A relatively flat area of skull is chosen, taking care to avoid the mastoid air cells, the mastoid emissary vein, or the lateral or sigmoid sinus. This part of the cranium behind the ear lies within the sphere of natural awareness and avoids trauma to the connector.

For the first patient, we used 3 separate neck incisions passing across the midline to zigzag the cable for tension relief (Figure 5). We now consider the zigzag to be unnecessary and use one incision over the trapezius muscle on the posterior aspect of the neck to provide a gentle curve between the thoracic exit and the skull pedestal.

\section{Surgical Procedure}

The patients were brought to the intensive care unit on the evening before the operation, where a continuous cardiac output monitoring system and arterial line were inserted. An infusion of the inodilator milrinone was started. The following morning, after induction of anesthesia, a left-sided double-lumen endotracheal tube was inserted, and a transesophageal echocardiography probe was deployed. The patient was positioned in the lateral position, providing access to the scalp, neck, left chest, and left femoral vessels. Detailed hemodynamic control was maintained with a combination of nitric oxide gas to reduce pulmonary arterial pressure and glyceryl trinitrin, esmolol, adrenalin, and dopamine infusions. We aim to complete the implantation of the power-delivery system before heparinization. This requires about 1 hour, with the patient in the left lateral position before beginning partial cardiopulmonary bypass.

The operation began with simultaneous exposure of the parietal bone and femoral vessels by different teams. In the event of profound hemodynamic deterioration, heparinization, cannulation, and cardiopulmonary bypass were then possible. The skull pedes- 

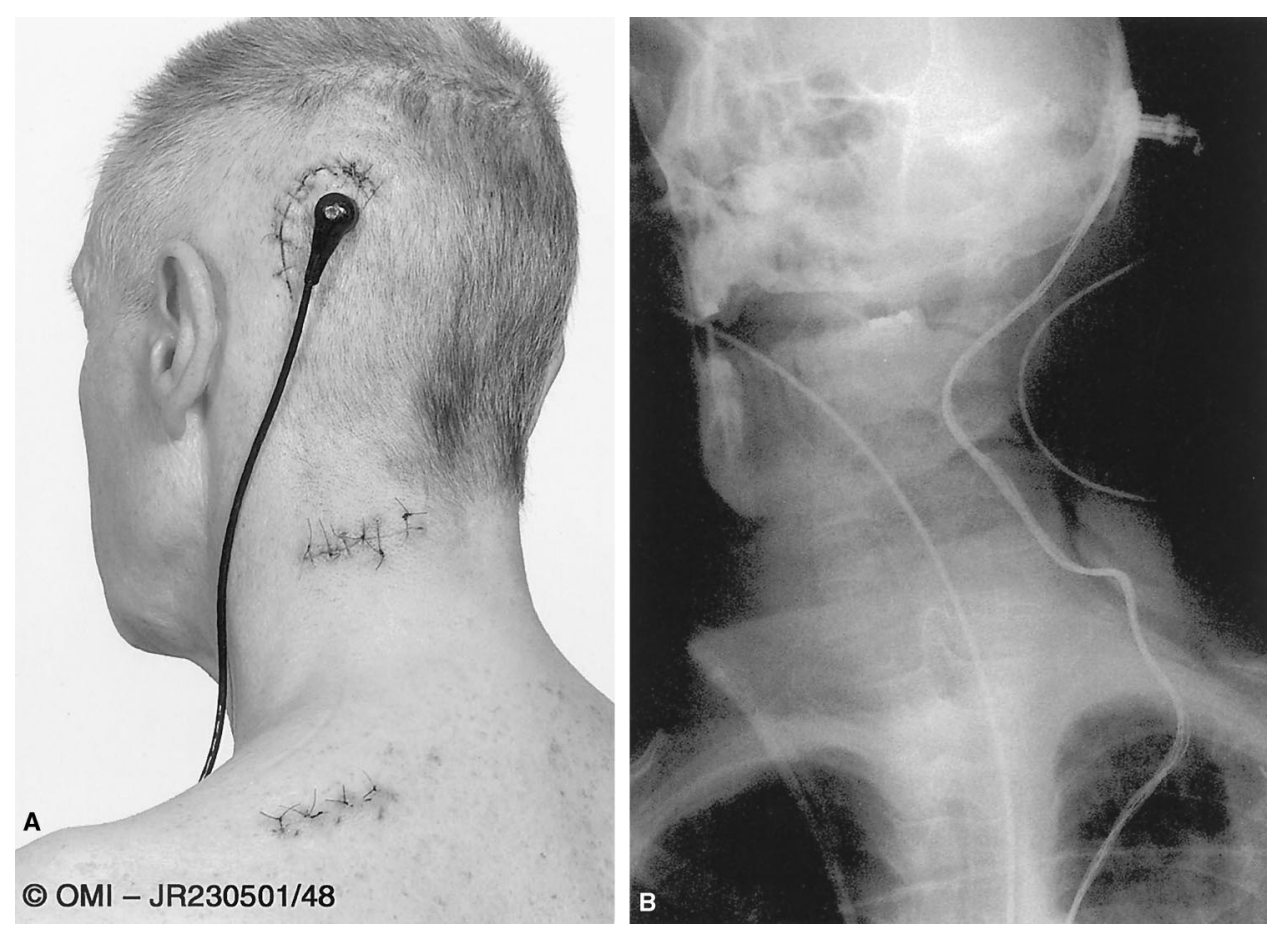

Figure 2. Passage of the power cable from the second intercostal space to the postauricular pedestal: A, skin incision and external connector; B, plain radiograph showing the power cable passing in a gentle curve through the neck.

tal was implanted by an experienced cochlear implant surgeon (A.F.). A cork bore-type instrument the same diameter as the percutaneous part of the titanium pedestal was used to tunnel through the skin to the periosteum at the chosen site. An appropriately sized skin defect was thereby created. A C-shaped incision was then made around this on the basis of the occipital arterial supply, and a full-thickness flap was raised down to the periostium (Figure 2, A). The periostium was elevated beneath the skin flap, and a template was used to define the position of the bone screws. Any skull irregularity was burred off to give a flat surface. A dental drill was used to penetrate the external table before inserting the self-tapping screws.

Meanwhile, the left thoracotomy was performed through the sixth intercostal space to expose the apex of the left ventricle and the descending thoracic aorta. The Jarvik 2000 heart and power cable were brought into the surgical field, and the incisions were made on the shoulder and neck to convey the 3-pin connector and power cable to the skull-pedestal site. This was achieved by inserting the 3-pin connector within the end of an intercostal drain. The drain was withdrawn out of the chest and through the neck to the scalp. The 3-pin connector was then inserted through the titanium pedestal (Figure 3, A). This was then implanted firmly onto the external table by using 7- or 8-mm bone screws (Figure 3, $B$ ). Bone dust from flattening of the external table or the screw hole was used to promote osseointegration. The postauricular skin flap was repositioned with the percutaneous pedestal through the punched-out defect. The scalp and skin incisions were then closed securely. The external power cable was attached to the skull pedestal, and with the Jarvik 2000 heart in a bowl of saline, the power was switched on to test the circuit.
A side clamp was then applied to the descending thoracic aorta, to which a 16-mm Dacron graft was anastomosed. Systemic heparinization was then undertaken, and the left femoral artery and vein were cannulated for cardiopulmonary bypass.

The pericardium was opened widely anterior to the left phrenic nerve. The apex of the globular left ventricle was identified and cored, and the Jarvik 2000 restraining cuff was sewn into position. The Jarvik 2000 heart was then inserted through the cuff and fixed in position with circumferential tapes. The Dacron graft attached to the pump was trimmed to length and anastomosed to the graft on the descending aorta. Meanwhile, the heart was defibrillated and filled to dispel air. When the whole system was thoroughly deaired, the pump was switched on, and the patient was weaned from cardiopulmonary bypass.

\section{Postoperative Care}

The percutaneous pedestal required very little maintenance. ${ }^{4}$ On the first postoperative day, the dressings were removed, and the site was cleaned and kept dry. The external cable was disconnected, and blood clot or debris was cleaned from the surfaces. The patients tolerated discontinuation of pump flow for up to $5 \mathrm{~min}$ utes, with a functional aortic regurgitation flow of 800 to 1000 $\mathrm{mL} / \mathrm{min}$. For the first 3 months during the process of osseointegration, every attempt was made not to apply weight or tension to the pedestal.

\section{Results}

During the learning curve for the procedure, implantation of the power system took between 50 and 80 minutes. For the 

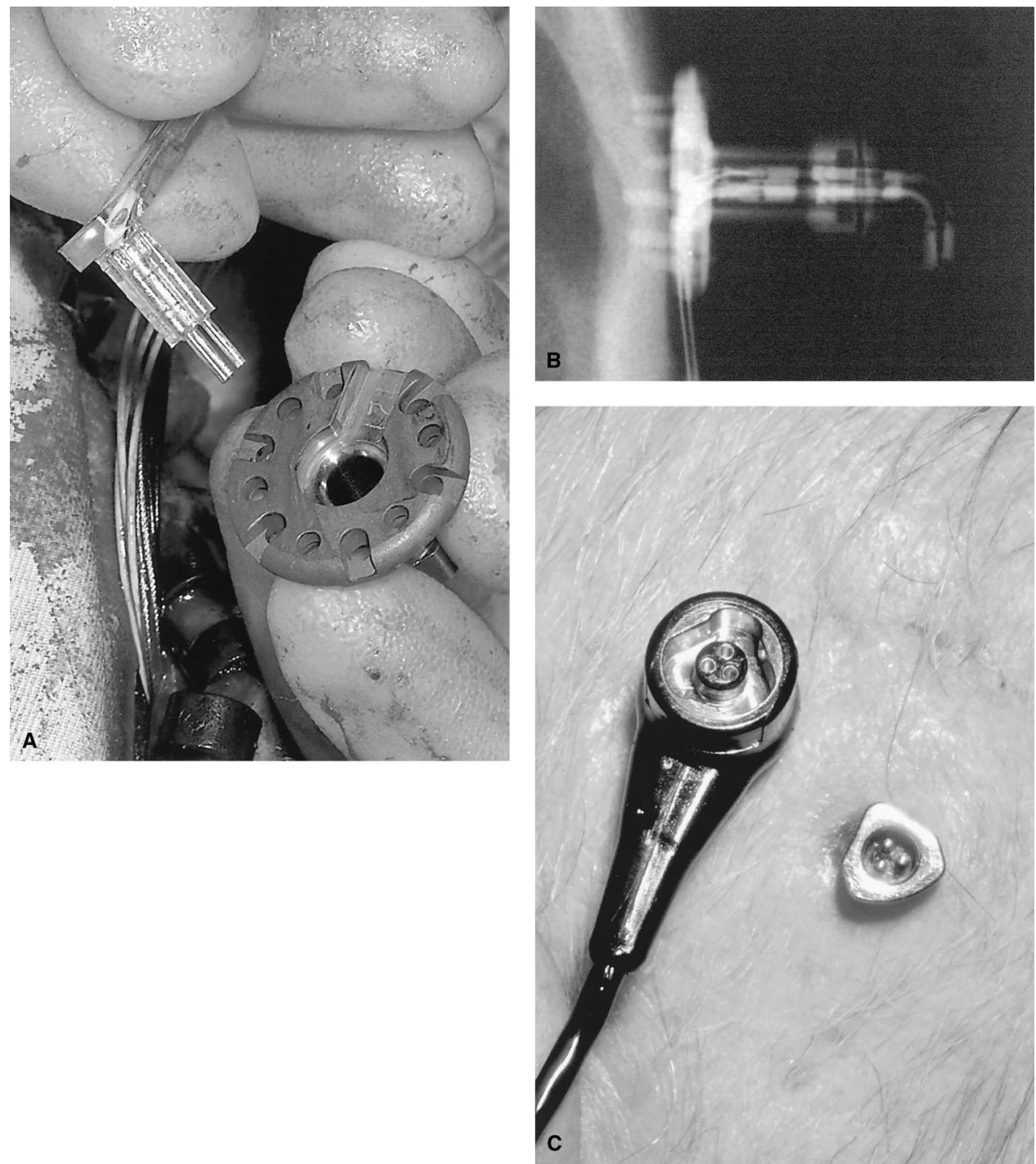

Figure 3. Components of the percutaneous system: A, 3-pin connector and titanium pedestal; B, pedestal screwed into the skull; C, healing pedestal with external connector.

first patient, access to both sides of the neck to zigzag the cable proved difficult, and profound hemodynamic deterioration occurred when the left lung was collapsed. This required early heparinization and cardiopulmonary bypass before the system was finished. During the second postoperative week, an acute bend in the cable began to erode through one of the neck incisions. This required revision into a deeper pocket by using local anesthetic and a regional nerve block. After this, all wounds healed satisfactorily, and there has been no infection over 12 months. The patient lived at home in New York Heart Association class I for 10 months and has now survived 1 year after implantation.

In the second $(112 \mathrm{~kg})$ patient the implant seemed uneventful, and he initially recovered from anesthesia and moved all limbs to command. However, severe coagulopathy resulted in excessive blood transfusion with pulmonary hypertension and the need for prolonged sedation and positive-pressure ventilation. Ten days after the operation, he was anticoagulated with warfarin, but on discontinuing sedation, it was apparent that there was right-sided weakness. A CT scan revealed a subdural hemorrhage adjacent to the site of the pedestal (Figure 6). Craniotomy was performed, and the pedestal was detached and repositioned in a subcutaneous pocket. Warfarin was discontinued. The site healed well, but because of his size and neurologic impairment, it proved difficult to wean him from the ventilator. The combination of positive-pressure ventilation and pulmonary hypertension exacerbated tricuspid regurgitation and right heart failure. Although the pump continued to function 


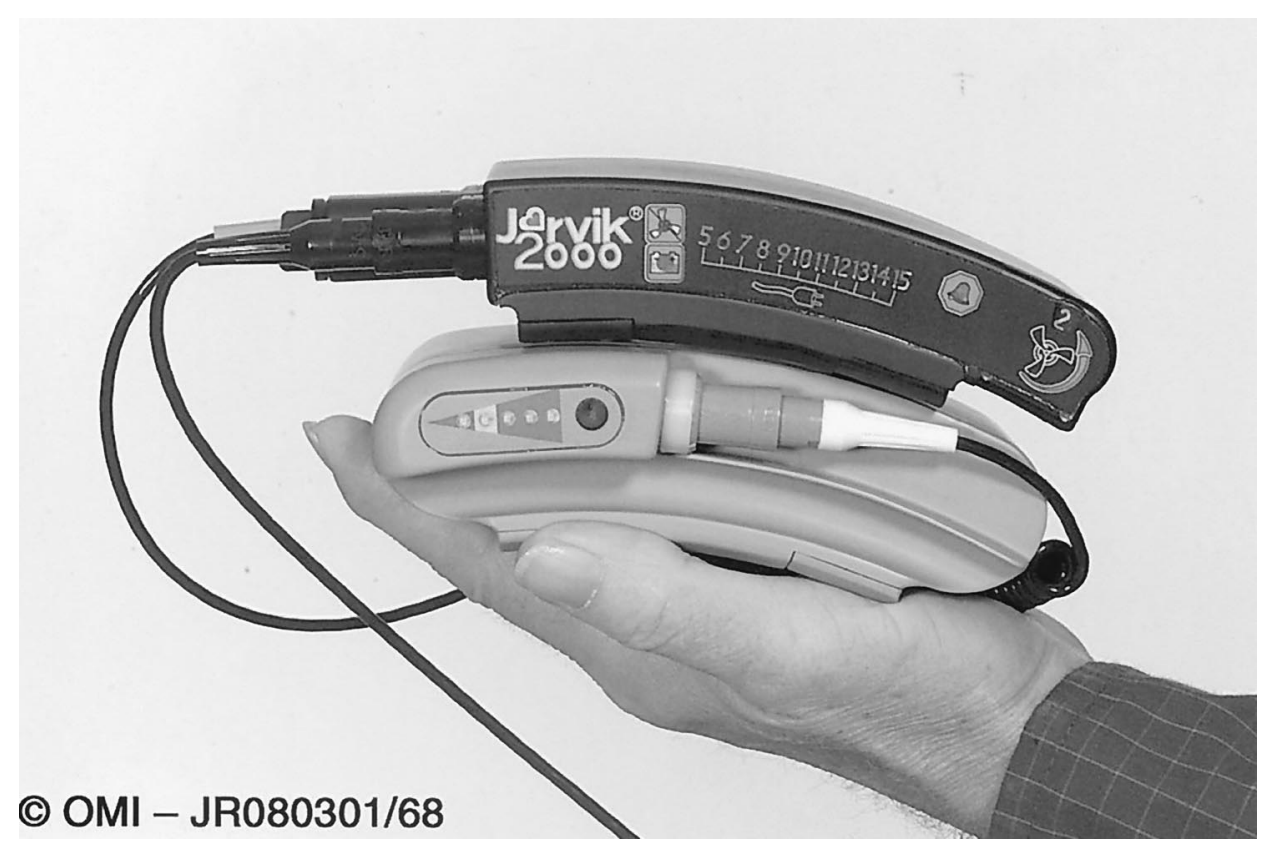

Figure 4. The controller and portable battery (combined weight $<1 \mathrm{~kg}$ ).

satisfactorily without anticoagulation, the patient died 3 months after implantation.

In patients 3 and 4 the postauricular pedestal healed well and remains free from infection 4 weeks and 4 months later. The patients were discharged home 3 weeks after the implantation.

The system is user friendly in the outpatient setting. The patients are able to shower and wash the hair normally. The pedestal site is checked and cleaned thoroughly by the LVAD nurse on outpatient visits. When the hair regrows, the external system is virtually invisible, passing from behind the ear and then under the shirt or jacket. The system is robust, and all external components can be replaced. Our first patient carries both controller and battery in a shoulder bag. He has carried his system overseas by air for a vacation. On one occasion, a thief snatched the bag outside a supermarket. The connecting cable was avulsed from the pedestal and activated the alarm. This caused the thief to drop the bag, after which the cable was reconnected. On another occasion, the controller and battery were dropped onto a hard surface, which damaged the cable connection. Again, he was able to exchange the cable and restarted the pump 20 minutes later without adverse effects.

\section{Discussion}

The pusher-plate LVADs are large space-occupying foreign bodies with a direct connection to the skin through the driveline. Now patients undergoing LVAD implantation are more frequently released into the community, and the incidence of late infective complications is prohibitive. Positive driveline cultures have been recorded in $50 \%$ of patients successfully bridged to transplantation, with obvious clinical infection in $30 \% . .^{7,8}$ Currently, about $30 \%$ of patients are ultimately rejected for transplantation because of infection, and $29 \%$ of those with major driveline problems die while waiting for a donor organ. ${ }^{9}$ Staphylococcal organisms are responsible for the majority of percutaneous site infections. Disseminated Candida species infection has been described in $28 \%$ of patients undergoing Thermo Cardiosystems HeartMate LVAD implantation (Thermo Cardiosystems, Inc, Woburn, Mass) by 3 months after implantation and in $45 \%$ by 9 months. ${ }^{10}$ This appears to be due to progressive defects in T-cell immunity. Although infection may be related to the patient's overall clinical condition, the thick, stiff driveline attached to the bulky mobile LVAD and penetrating mobile skin contributes to the risk. Both localized and ascending driveline infections are usually preceded by nonhealing of the exit site, where the rigid cable moves with the patient's activity. Infection generally leads to rehospitalization, septic embolism, and reoperations, including revision of the percutaneous driveline or removal or exchange of the infected LVAD. These problems have a negative effect on quality of life and cost of LVAD treatment. The Jarvik 2000 power line is of much smaller caliber and is not subject to the same stress as with pusher-plate cables. It is likely that these improvements alone will lead to fewer infective complications. Only 2 of 10 bridge-to-transplantation patients in the Texas Heart Institute had superficial skin infection, and this did not alter their clinical course.

Bone-anchored implants that penetrate the epithelium have been successfully used for hearing aids and for the attachment of cosmetic prostheses in auricular malforma- 


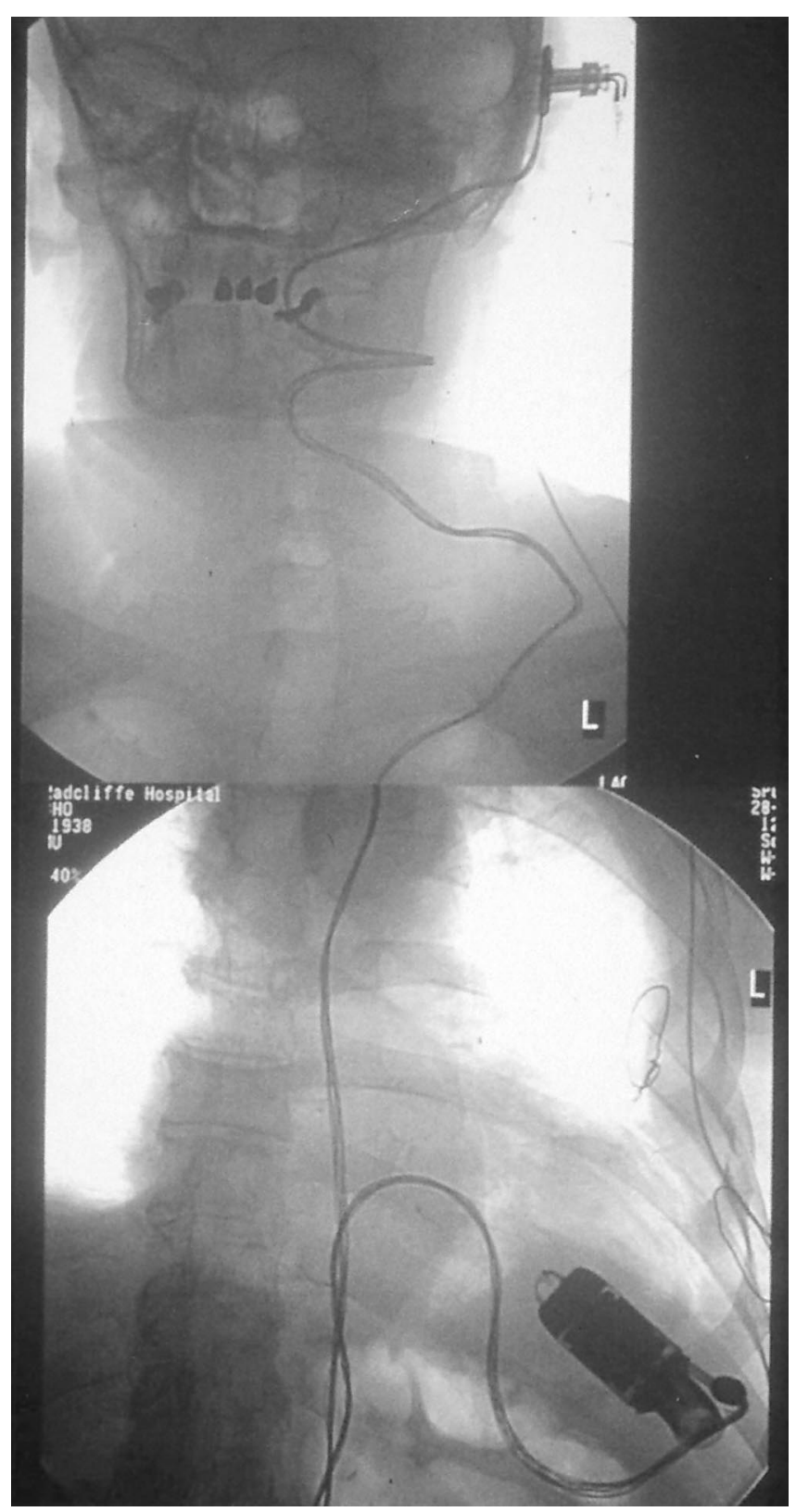

Figure 5. The Jarvik 2000 heart and power cable in patient 1 . The cable takes a zigzag path through the neck to prevent tension in the wires.

tions. ${ }^{4,11}$ When the soft tissues adhere tightly to the titanium stem, the risk of cutaneous bacteria reaching the pedestal base and causing infection is small. The biologic acceptance of bone-anchored foreign-body implants is influenced by many factors, including bone density, fixing screw, hosttissue reaction, and biocompatibility of the implant material. ${ }^{12}$ Osseointegration is the direct structural and functional connection between living bone and the surface of the load-carrying implant. In cochlear implants with a percutaneous button screwed to the skull, there is freedom from infection of greater than $90 \%$ at 10 years. ${ }^{11}$ Many patients have had an implant without infection for longer than 20 years. Consequently, the chances of infection reaching the Jarvik 2000 heart through the cable are virtually nil.

Our early clinical experience is encouraging. The pedestal and connector are only a little more obtrusive than an old-fashioned hearing aid. The patient cannot hear or feel the Jarvik 2000 heart within the chest, and the power system allows normal life in the community. Our implant learning curve has been important and allowed others to begin using the system without complications. We soon abandoned the zigzag method, which was time consuming, difficult to keep sterile, and unnecessary. The gentle curve of the cable with only one incision between the thoracic exit and the pedestal is simpler and safe.

Penetration of the inner table of the skull in the second patient was an avoidable complication. Surgical exploration showed a single shallow drill hole that would not have resulted in significant bleeding in the absence of coagulopathy or anticoagulation. We responded to this complication by changing the type of drill used and carefully measuring the thickness of the skull by means of CT scanning and having a variety of different-length self-tapping titanium screws available. Alternative bone-anchoring points, such as the sternum, a rib, or the iliac crest site, may also be feasible, but the depth of subcutaneous fat is unpredictable in these areas and may undermine the benefit of the scalp site.

For permanent implants, the ability of the patient to change the external system is a distinct advantage. Discontinuation of the power supply for up to 30 minutes has been tolerated, and the degree of functional aortic regurgitation (retrogradely through the vascular graft) seems to be less than for other devices. These patients already had significant improvement in left ventricular function (increase in myocardial oxygen consumption from $5.7 \mathrm{~mL} \cdot \mathrm{min}^{-1} \cdot \mathrm{kg}^{-1}$ preoperatively to $12.0 \mathrm{~mL} \cdot \mathrm{min}^{-1} \cdot \mathrm{kg}^{-1}$ in patient 1 ). Significantly, there was no thromboembolism on restoration of pump flow.

In summary, the clinical experience to 1 year echos our freedom from infection in the laboratory. Postauricular power delivery is user friendly in the outpatient setting, and to date, we have had no sepsis. Permanent mechanical circulatory support is feasible only with a mechanically reliable pump and an acceptably low risk of infection. The Jarvik 2000 heart with postauricular power delivery is promising in both respects.

Roger Grey, FRCS, and Stephen Watt-Smith, FRCS, provided invaluable advice regarding the surgical aspects of pedestal implantation. Patients 3 and 4 were operated on at the Heart Hospital, London, United Kingdom. We greatly appreciate the support provided by this institution. 


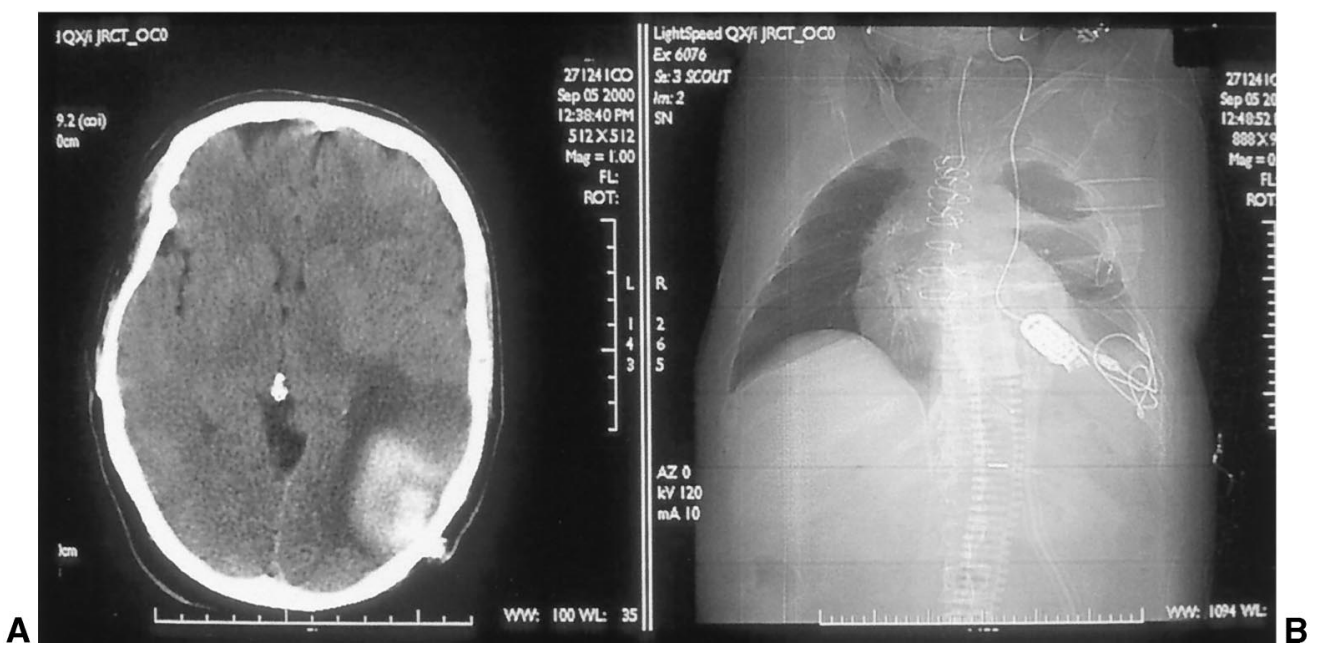

Figure 6. A, CT scan of the brain showing a subcutaneous hematoma adjacent to the pedestal. B, Plain chest radiograph showing the pump in the apex of the left ventricle.

\section{References}

1. Myers $\mathrm{T}$, Khan $\mathrm{T}$, Frazier OH. Infectious complications associated with ventricular assist system. ASAIO J. 2000;46:S28-36.

2. Griffith BP, Kormos RL, Nastala CJ, Winowich S, Pristasis JM. Results of extended bridge to transplantation: window into the future of permanent ventricular assist device. Ann Thorac Surg. 1996;61: 396-8.

3. Mussivand T, Miller JA, Santerre PJ, Belanger G, Rajagopalan KC, Hendry PJ, et al. Transcutaneous energy transfer system performance evaluation. Artif Organs. 1993;17:940-7.

4. Parkin JL. Percutaneous pedestal in cochlear implantation. Ann Othol Rhinol Laryngol. 1990;99:796-800.

5. Jarvik R, Westaby S, Katsumata T, Pigott D, Evans RD. LVAD power delivery: a percutaneous approach to avoid infection. Ann Thorac Surg. 1998;65:470-3.

6. Westaby S, Katsumata T, Houel R, Pigott D, Frazier OH, Jarvik RK. Jarvik 2000 Heart; potential for bridge to myocyte recovery. Circulation. 1998;98:1568-74.
7. Springer WE, Wasler A, Radovancevic B, Myers T, Odegaard P, Macris MP. Retrospective analysis of infection in patient undergoing support with left ventricular assist device. ASAIO J. 1996;42:763-5.

8. Fischer SA, Trenholmer GM, Costanzo MR, Piccione W. Infectious complications in left ventricular assist device recipients. Clin Infect Dis. 1997;24:18-23.

9. Ankersmit J, Tugulea S, Sparier T, Weinberg AD, Atrip JH, Buske EM, et al. Activation induced $\mathrm{T}$ cell death and immune dysfunction after implantation of a left ventricular assist device. Lancet. 1999;354:550-5.

10. Herrmann M, Weyand M, Greshake B, von Eiff C, Proctor RA, Scheld $\mathrm{HH}$, et al. Left ventricular assist device infection is associated with increased mortality but is not a contraindication to transplantation. Circulation. 1997;95:814-7.

11. Van de Heyning PH, D'Haese PS, Claes J, Koekelkoren E, De Bodt M. Cochlear implantation: an over view of existing implants. Acta Otorlaryngol Belg. 1998;52:91-103.

12. Kohri M, Cooper EP, Ferracane JL, Waite DF. Comparative study of hydroxyapatite and titanium dental implants in dogs. J Oral Maxillofac Surg. 1990;48:1265-73. 